\title{
Space Mapping Design Framework Exploiting Tuning Elements
}

\author{
Qingsha S. Cheng, Senior Member, IEEE, John W. Bandler, Life Fellow, IEEE, and
} Slawomir Koziel, Senior Member, IEEE

\begin{abstract}
Inspired by the ideas of "simulator-based" tuning, implicit space mapping, and surrogate optimization, we propose an implementable microwave design framework. In this framework, we alter an electromagnetic (EM) model by embedding suitable tuning elements. The resulting tuning model is aligned with the original unaltered EM model. We then designate the aligned tuning model as surrogate for design optimization purposes. We illustrate our tuning space mapping framework using a simple microstrip line example. Several microwave examples, including a low-temperature co-fired ceramic filter demonstrate the framework's implementation and robustness.
\end{abstract}

Index Terms-Computer-aided design (CAD), design tuning, electromagnetic (EM)-based design, engineering optimization, space mapping, surrogate models.

\section{INTRODUCTION}

$\mathbf{E}$ LECTROMAGNETIC (EM) simulation is accurate, but CPU intensive; hence, using a full-wave EM simulator to optimize a complex structure may be prohibitive. An approach is to use decomposition, i.e., to break down an EM-based model and combine the components within a circuit simulator in the hope of reducing CPU demand. Co-simulation or co-optimization of EM/circuits [1]-[7] is a common industry solution to blend EM-simulated components into circuit models. These methods embed parameterized EM or interpolated EM components in a circuit simulator to form a surrogate for optimization. Swanson et al. [8], [9] and Rautio [10] propose a "simulator-based" tuning method in which circuit-theory-based tunable elements are embedded in an EM simulator through internal ports created in the EM model. The resulting surrogate can be optimized.

Manuscript received May 13, 2009. First published December 15, 2009; current version published January 13, 2010. This work was supported in part by the Natural Sciences and Engineering Research Council of Canada under Grant RGPIN7239-06 and Grant STPGP336760-06, by Bandler Corporation, and by the Reykjavik University Development Fund under Grant T09009.

Q. S. Cheng is with the Simulation Optimization Systems Research Laboratory, Department of Electrical and Computer Engineering, McMaster University, Hamilton, ON, Canada L8S 4K1 (e-mail: chengq@ mcmaster.ca).

J. W. Bandler is with the Simulation Optimization Systems Research Laboratory, Department of Electrical and Computer Engineering, McMaster University, Hamilton, ON, Canada L8S 4K1, and also with Bandler Corporation, Dundas, ON, Canada L9H 5E7 (e-mail: bandler@mcmaster.ca).

S. Koziel is with the Engineering Optimization and Modeling Center, School of Science and Engineering, Reykjavík University, IS-103 Reykjavík, Iceland (e-mail: koziel@ru.is).

Color versions of one or more of the figures in this paper are available online at http://ieeexplore.iee.org.

Digital Object Identifier 10.1109/TMTT.2009.2036395
In this paper, we extend the idea of "simulator-based" tuning and discuss some variations and combine them into one framework. We define multiple auxiliary ports within a structure, e.g., in the manner of the "co-calibrated" [11] internal ports of Sonnet $\boldsymbol{e m}^{1}$ and the un-calibrated internal ports of Agilent Momentum. ${ }^{2}$ Elements can then be incorporated into the structure using such ports. EM-simulator-based tuning techniques [9], [12]-[14] apply tunable (tuning) elements across or between these internal ports [10], [15]. The resulting model (which we call "tuning model") becomes a surrogate for design or modeling purposes in the context of tuning space mapping [12]-[14].

We define two ways of embedding the tuning elements into the EM model: we call them "Type 0" and "Type 1." By "Type 0 " embedding we refer to inserting tuning elements across "infinitesimal" gaps between the internal ports, as in [12] and [13]. The so-called "Type 1" embedding refers to replacing an entire section of design interest between the internal ports with a tuning element. In [14], the tuning model is formed using Type 1 embedding. The embedded tuning elements are normally selected from equivalent-circuit components available in a circuit simulator.

These tuning elements can be further divided into two categories, "direct" and "indirect." Direct tuning elements are equivalent-circuit substitutes with parameters consistent with the original EM model parameters, e.g., an equivalent circuit of a microstrip line. Indirect tuning elements are equivalent circuits usually with a set of (indirect) tuning parameters perhaps different than its EM counterpart, e.g., an ideal transmission line specified by electrical length and characteristic impedance.

Type 0 embedding as described in [12] and [13] has minimal disturbance to the EM model. However, it has a limited tuning capacity. At times, negative valued tuning elements may be required since they are initialized as zero. Although not a deterrent to Agilent $\mathrm{ADS}^{3}$ this might pose difficulties to circuit simulators. Often an indirect tuning element has to be employed to represent the physical structure. The indirect tuning parameter values require translation to the physical design parameter values. Extra calibration steps are necessary to determine the translation formula [12], [13]. Furthermore, the tuning space mapping with Type 0 embedding presented in [12] and [13] does not easily handle certain cross-sectional parameters such as widths and substrate heights of a microstrip line.

\footnotetext{
${ }^{1}$ Sonnet $\boldsymbol{e m}$, version 12.54, Sonnet Software Inc., North Syracuse, NY, 2009.

${ }^{2}$ Agilent Momentum, version 2008U1, Agilent Technologies, Palo Alto, CA, 2008.

${ }^{3}$ Agilent ADS, version 2008U1, Agilent Technologies, Palo Alto, CA, 2008.
} 
Utilizing Type 1 embedding [14] in the tuning space mapping, we address these issues. Instead of inserting a tuning element into the gap between adjacent internal ports, we replace an entire section of design interest with a tuning element. Such tuning elements, likely equipped with direct and positive tuning parameter values, are able to model cross-sectional parameters. Replacing large sections of the original structure also allows significant tuning capacity. Couplings among and within the remaining structure are maintained. With suitable adjustments of the relevant tuning element, the responses of the resulting tuning model can match those of the original structure.

With Type 1 embedding, a large number of equivalent circuits with direct tuning parameters (such as coupled microstrip line) in the component library of the circuit simulator become useful. Therefore, we could rely less on indirect tuning elements than for Type 0 embedding. However, our new technique allows such indirect tuning elements to be embedded in the Type 1 tuning model. In this case, the required translation (or so-called calibration) of the indirect tuning element parameters to the design parameters is simpler than for Type 0 embedding, as described in [12] and [13].

In this paper, we propose a space mapping design framework that is capable of dealing with Type 0 and/or Type 1 embedding and direct and/or indirect tuning elements.

\section{Tuning Space Mapping Framework}

Space mapping [16], [17] shifts the CPU burden from an expensive EM simulator (fine model) to a fast equivalent-circuit model (coarse model). We consider simulation-based tuning [8], [10] within the scope of space mapping [13]. In our tuning space mapping approach, a tuning model of the fine model is constructed by either attaching tuning elements to internal ports or replacing portions of designable interest between ports by suitable tuning elements. The parameters and/or preassigned parameters of these elements are chosen to be tunable. In each iteration, the tuning model is matched to the fine model by optimizing the preassigned parameters or/and the tuning parameters. This process takes little CPU effort as the tuning model is typically implemented within a circuit simulator. With optimal preassigned parameters or/and the tuning parameters thus obtained, an updated tuning model is available for design prediction. The prediction is fed back to fine model simulator after simple calibration. This process is repeated until the fine model response is sufficiently close to the design target.

\section{Tuning Space Mapping Algorithm}

We are concerned with the optimization problem

$$
\boldsymbol{x}_{f}^{*}=\arg \min _{\boldsymbol{x}} U\left(\boldsymbol{R}_{f}(\boldsymbol{x})\right)
$$

where $\boldsymbol{R}_{f} \in R^{m}$ denotes the response vector of a fine model of the device of interest, $U$ is a merit function (e.g., a minimax function or a norm), $\boldsymbol{x}$ is a vector of design parameters, and $\boldsymbol{x}_{f}^{*}$ is the optimal solution to be determined.

In [12] and [13], tuning space mapping involves Type 0 embedding in which tuning elements are attached to internal ports of the fine model [10] [see Fig. 1(a)]. If a tuning-to-design-parameter conversion is not available, for calibration

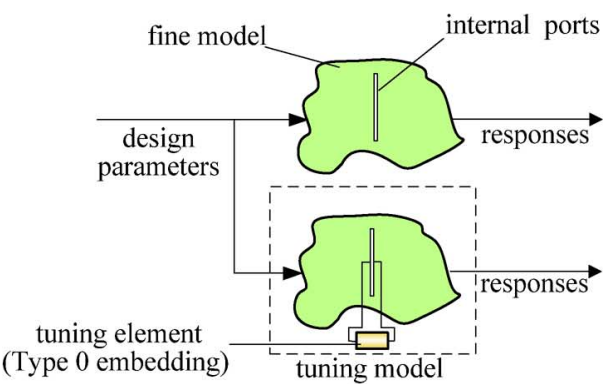

(a)

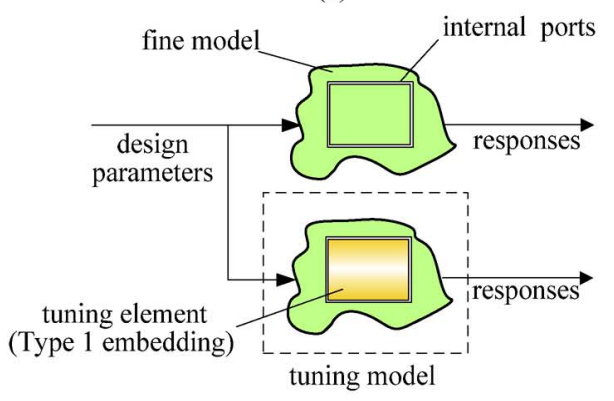

(b)

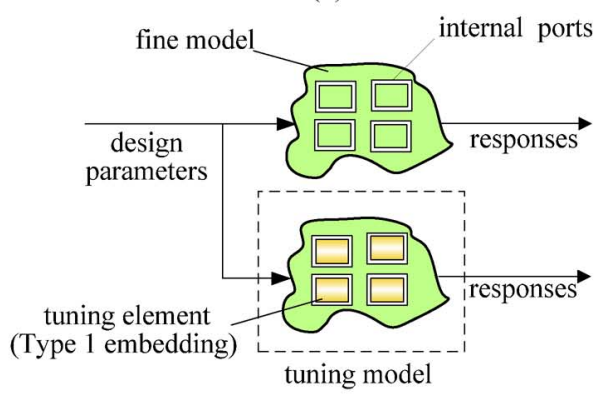

(c)

Fig. 1. Tuning space mapping and tuning model. (a) Type 0 embedding. (b) Type 1 embedding of a single tuning element. (c) Type 1 embedding of multiple tuning elements. Type 0 embedding normally involves only one cut and one port on each side of the cut in the fine model. Type 1 embedding usually involves two or more cuts in the fine model, and the number of ports is twice the number of cuts.

purposes we require an additional full-system coarse model or the simulation of a perturbed fine model. For simplicity, our present framework excludes the method that requires the additional full-system coarse model. In [14], we replace certain designable sub-section(s) with suitable Type 1 embedding [see Fig. 1(b) and (c)]. In most of our examples, they have direct tuning parameters, i.e., distributed microwave circuit elements with physical dimensions corresponding to those of the fine model. In a simple parameter extraction procedure, or, rather, an alignment procedure, we match the tuning model with the fine model (the original structure without internal ports). We assume that certain fine-model couplings are preserved in the tuning model. Thereby we normally obtain a good surrogate of the fine model that is subsequently tuned to satisfy our goals by changing its tuning parameters. The obtained tuning parameter values are converted to design parameter values that become our next fine model iterate (design).

Our algorithm produces a sequence of points (design variable vectors) $\boldsymbol{x}^{(i)}, i=0,1, \ldots$ The iteration of the algorithm consists of three steps, which are the: 1) alignment of the tuning 
TABLE I

COMPARISON OF TUNING MODELS

\begin{tabular}{llll}
\hline tuning model & $\begin{array}{l}\text { embedding } \\
\text { method }\end{array}$ & $\begin{array}{l}\text { parameters } \\
\text { extracted to } \\
\text { match to fine } \\
\text { model }\end{array}$ & $\begin{array}{l}\text { tuning element } \\
\text { type }\end{array}$ \\
\hline & Type 0 & $\boldsymbol{t}_{0}$ & direct \\
& Type 1 & $\boldsymbol{t}_{0}$ or $x_{p}$ & direct \\
& $\boldsymbol{t}_{0}$ & indirect \\
\hline$x_{1}$ & Type 0 & $\boldsymbol{t}_{0}$ & indirect
\end{tabular}

model with the fine model; 2) optimization of the tuning model; and 3) calibration of the tuning parameters to the design parameters.

In our first step, based on data from the fine model (with internal ports) at point $\boldsymbol{x}^{(i)}$, the current tuning model $\boldsymbol{R}_{t}^{(i)}$ is built with appropriate Type 0 or Type 1 embedding. The tuning model response may not agree with the response of the original fine model $\boldsymbol{R}_{f}$ at $\boldsymbol{x}^{(i)}$. We align these models by the following procedure [17]:

$$
\left(\boldsymbol{x}_{p}^{(i)}, \boldsymbol{t}_{0}^{(i)}\right)=\arg \min _{\boldsymbol{x}_{p}, \boldsymbol{t}_{0}}\left\|\boldsymbol{R}_{f}\left(\boldsymbol{x}^{(i)}\right)-\boldsymbol{R}_{t}^{(i)}\left(\boldsymbol{t}_{0}, \boldsymbol{x}_{p}\right)\right\|
$$

where $\boldsymbol{R}_{t} \in R^{m}$ denotes the response vector of the tuning model, $\boldsymbol{x}_{p}$ represents the preassigned parameters of the tuning model, and $\boldsymbol{t}_{0}$ represents the initial tuning parameters of the tuning model. We may only need to extract either $\boldsymbol{t}_{0}$ or $\boldsymbol{x}_{p}$ depending on the type of tuning model. We compare different tuning models in Table I.

In the second step, we optimize $\boldsymbol{R}_{t}^{(i)}$ to have it meet the design specifications. We obtain the optimal values of the tuning parameter $\boldsymbol{t}_{1}^{(i)}$ as

$$
\boldsymbol{t}_{1}^{(i)}=\arg \min _{\boldsymbol{t}_{1}} U\left(\boldsymbol{R}_{t}^{(i)}\left(\boldsymbol{t}_{1}, \boldsymbol{x}_{p}^{(i)}\right)\right) .
$$

Having $\boldsymbol{t}_{1}^{(i)}$, we perform the calibration procedure to determine the values of the design variables that yield the same change in the tuning model response as $\boldsymbol{t}_{0}^{(i)}$ goes to $\boldsymbol{t}_{1}^{(i)}$.

The new design is obtained through the calibration step [13]

$$
x^{(i+1)}=x^{(i)}+s^{(i)} *\left(t_{1}^{(i)}-t_{0}^{(i)}\right)
$$

where $\boldsymbol{s}^{(i)}$ is a real vector and $*$ denotes a component-wise multiplication. For direct tuning elements, $\boldsymbol{s}^{(i)}=\left[\begin{array}{llll}1 & 1 & \ldots & 1\end{array}\right]^{T}$. Otherwise a calibration step follows to calculate $\boldsymbol{s}^{(i)}$.

Step 3 can be realized in various ways by calibration [13]. We discuss two simple possibilities here. A complex calibration that involves a full coarse model is described in detail in [13].

\section{A. Direct Calibration}

This is the simplest calibration method where the relation between the design variables and the parameterized tuning parameters is assumed to be an identity function, i.e., we have $\boldsymbol{x}^{(i+1)}=\boldsymbol{x}^{(i)}+\left(\boldsymbol{t}_{1}^{(i)}-\boldsymbol{t}_{0}^{(i)}\right)$. This calibration method is used if the tuning components are directly related to the elements of the microwave structure represented by the design variables

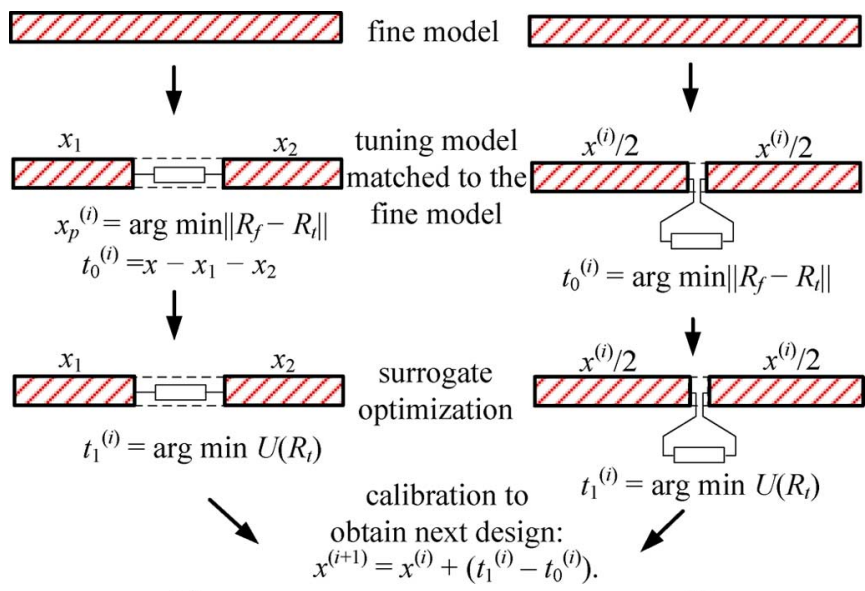

(a)

(b)

Fig. 2. Tuning space mapping with direct calibration of: (a) Type 1 embedding and (b) Type 0 embedding of parameterized tuning elements.

(e.g., microstrip line models as tuning elements and actual microstrip line lengths as design variables). We demonstrate the tuning space mapping process with direct calibration in Fig. 2 using a 1-D microstrip line design example.

\section{B. Analytical Calibration}

Analytical calibration deals with an indirect tuning parameter. In certain cases there exists an explicit analytical relation between the design variables and the non-parameterized tuning parameters so that (4) is replaced simply by applying this relation. In most cases, the calibration formula may be a linear function as in (4). We show a 1-D example in Fig. 3. For the Type 1 tuning model, $s$ is obtained by dividing the extracted tuning parameter $t_{0}^{(i)}$ by the physical dimension of the replaced section, as shown in Fig. 3(a). For the Type 0 tuning model, $s$ is obtained by dividing the (extracted) tuning parameter value difference by the design parameter value difference of the fine model before and after perturbation [see Fig. 3(b)].

\section{ILLUSTRATIONS AND EXAMPLES}

To illustrate and clarify our tuning space mapping algorithm, we use a microstrip transmission line [13]. The fine model is implemented in Sonnet $\boldsymbol{e m}$ (Fig. 4) and the fine model response is the inductance of the line as a function of the line's length. The original length of the line is chosen to be $\boldsymbol{x}^{(0)}=10.16 \mathrm{~mm}$ (400 mil) with a width of $0.635 \mathrm{~mm}$. We use a cell size of $0.01 \times 0.635 \mathrm{~mm}$. A substrate with thickness $H=0.635 \mathrm{~mm}$ and $\varepsilon_{r}=9.8$ is used. Our goal is to find a length of line such that the corresponding inductance is $6.5 \mathrm{nH}$ at $300 \mathrm{MHz}$. The Sonnet $\boldsymbol{e m}$ simulation at $\boldsymbol{x}^{(0)}$ gives the value of $4.52 \mathrm{nH}$, i.e., $\boldsymbol{R}_{f}\left(\boldsymbol{x}^{(0)}\right)=4.52 \mathrm{nH}$.

We apply the tuning space mapping algorithm of Section III. We show that the tuning model $\boldsymbol{R}_{t}$ is developed by dividing the structure in Fig. 4 into two or three parts and adding the tuning ports at the edges of the separated parts, as shown in Fig. 5(a) and (b). A Type 0 tuning element (e.g., a small inductor or a microstrip line segment) can now be attached between ports 3 and 4 in Fig. 5(a). A Type 1 tuning element can replace the section between ports 3 and 6 in Fig. 5(b). Note that the new 


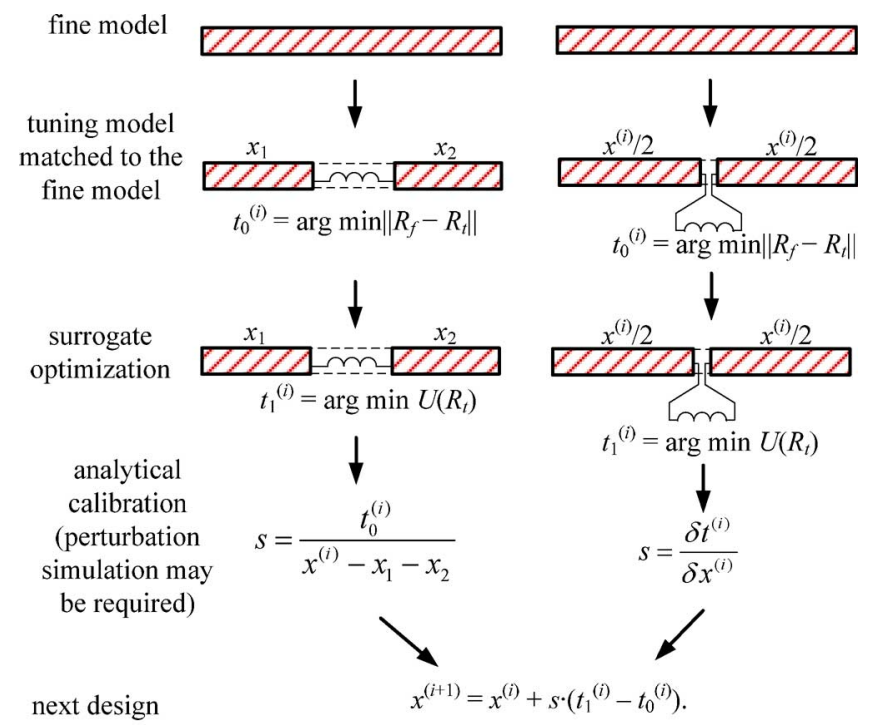

(a)

(b)

Fig. 3. Tuning space mapping with analytical calibration of: (a) Type 1 and (b) Type 0 tuning model, where $\delta t^{(i)}$ and $\delta x^{(i)}$ are value differences in the tuning and design parameters before and after perturbation.

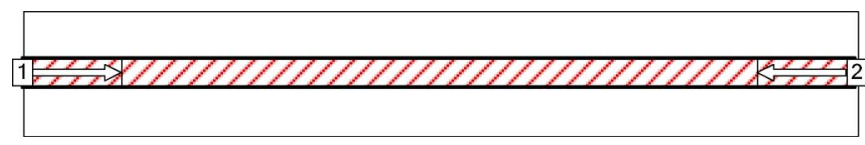

Fig. 4. Original structure of microstrip line in Sonnet.

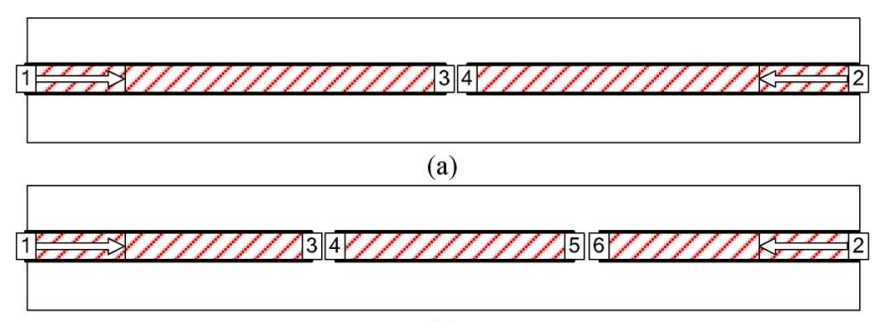

(b)

Fig. 5. Microstrip line under test after being cut, and with co-calibrated ports prepared for: (a) Type 0 tuning and (b) Type 1 tuning.

version of Sonnet $\boldsymbol{e m}$ allows so-called co-calibrated ports [11]. The ports allow an "infinitesimal" gap (normally one cell size wide) to be inserted into a microwave structure. A pair of such ports can then be mounted on the edges of the gap. The multiport structure is then simulated electromagnetically in Sonnet $\boldsymbol{e m}$ and connected in the circuit simulator. The partition and gaps are compensated by Sonnet $\boldsymbol{e m}$ so that the impact on the simulation results is negligible.

Type 0 Embedding With Direct Tuning Element: The tuning model is implemented in Agilent ADS and shown in Fig. 6(a). The model contains fine model data at the initial design in the form of an S4P element (" 4 " being the number of ports), as well as the attached tuning element (microstrip line segment). First, we find with $t_{0}^{(0)}=0.01 \mathrm{~mm}$ that the tuning model matches the fine model pretty well.

Next, we optimize the tuning model to meet our target of $6.5 \mathrm{nH}$. This yields $t_{1}^{(0)}=4.52 \mathrm{~mm}$.

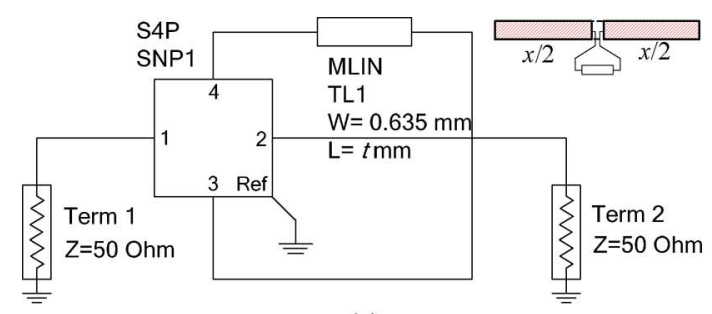

(a)

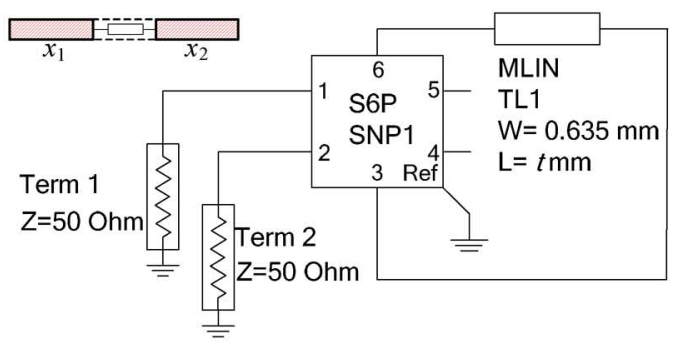

(b)

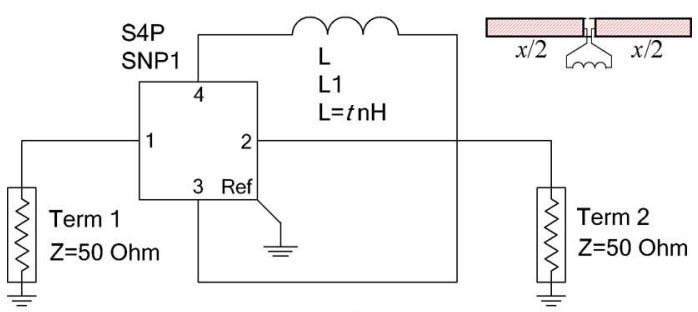

(c)

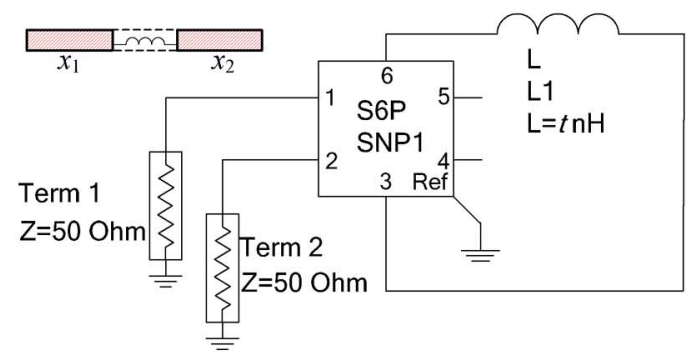

(d)

Fig. 6. Microstrip line design problem: tuning model and its ADS implementation. (a) Type 0 embedding with direct tuning element. (b) Type 1 embedding with direct tuning element. (c) Type 0 embedding with indirect tuning element. (d) Type 1 embedding with indirect tuning element.

Now, because the tuning element is a microstrip line segment, we use direct calibration, i.e., we assume that $x^{(1)}=$ $x^{(0)}+\left(t_{1}^{(0)}-t_{0}^{(0)}\right)$, which gives $x^{(1)}=14.67 \mathrm{~mm}$ and a corresponding fine model response of $6.59 \mathrm{nH}$. The second iteration gives $x^{(2)}=14.48 \mathrm{~mm}$, and the corresponding line inductance reaches our goal of $6.50 \mathrm{nH}$.

Type 1 Embedding With Direct Tuning Element: The model in Fig. 6(b) contains fine model data at the initial design in the form of an S6P element, as well as a Type 1 embedding of a direct tuning element (microstrip line segment). We start with $t_{0}=x^{(0)}-x_{1}-x_{2}=6.15 \mathrm{~mm}$ (here $x_{1}=x_{2}=2 \mathrm{~mm}$ ). After matching, $t_{0}=6.45 \mathrm{~mm}$. This initialization process compensates for errors introduced by the tuning elements and/or co-calibrated ports.

Next, we optimize the tuning model to meet our target $6.5 \mathrm{nH}$, which yields $t_{1}^{(0)}=10.96 \mathrm{~mm}$. Direct calibration gives $x^{(1)}=$ $14.66 \mathrm{~mm}$ and $6.58 \mathrm{nH}$. The second iteration yields $x^{(2)}=$ 
TABLE II

TRANSMISSION LINE ILLUSTRATION FIRST ITERATION SUMMARY

\begin{tabular}{|c|c|c|c|c|c|}
\hline tuning model & $x^{(0)}$ & $t_{0}^{(0)}$ & $t_{1}{ }^{(0)}$ & $s$ & $x^{(1)}$ \\
\hline \multirow{2}{*}{ 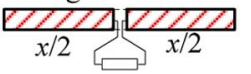 } & 10.16 & 0.01 & 4.52 & \multirow{2}{*}{1} & 14.67 \\
\hline & $\mathrm{mm}$ & $\mathrm{mm}$ & $\mathrm{mm}$ & & $\mathrm{mm}$ \\
\hline \multirow{2}{*}{$\frac{Z Z Z Z}{x_{1}}=\frac{E Z Z}{x_{2}}$} & 10.16 & 6.45 & 10.96 & \multirow{2}{*}{1} & 14.66 \\
\hline & $\mathrm{mm}$ & $\mathrm{mm}$ & $\mathrm{mm}$ & & $\mathrm{mm}$ \\
\hline \multirow{2}{*}{$\underset{x / 2}{\angle Z Z Z} x / 2$} & 10.16 & 0.00 & 1.94 & 2.249 & 14.51 \\
\hline & $\mathrm{mm}$ & $4 \mathrm{nH}$ & $\mathrm{nH}$ & $\mathrm{mm} \cdot \mathrm{nH}^{-1}$ & $\mathrm{~mm}$ \\
\hline \multirow{2}{*}{$\frac{\angle Z Z A}{x_{1}}$} & 10.16 & 2.74 & 4.7 & 3.14 & 14.56 \\
\hline & $\mathrm{mm}$ & $\mathrm{nH}$ & $\mathrm{nH}$ & $\mathrm{mm} \cdot \mathrm{nH}^{-1}$ & $\mathrm{~mm}$ \\
\hline & & 95 & & & \\
\hline$\overline{P 1} \mid \mathrm{IIIIIIP3} \leftrightarrow \mathrm{P} 5$ & IIII & $\#$ & $17+$ & & \\
\hline
\end{tabular}

Fig. 7. Coupled microstrip line Agilent Momentum model with internal ports.

$14.48 \mathrm{~mm}$. The corresponding line inductance reaches our goal of $6.50 \mathrm{nH}$.

Type 0 Embedding With Indirect Tuning Element: See Fig. 6(c). After initial matching, $t_{0}^{(0)}=0.004 \mathrm{nH}$. After optimization of the tuning model we have $t_{1}^{(0)}=1.94 \mathrm{nH}$.

For the calibration step, we propose a linear dependence between the microstrip length $x$ and the inductance of the tuning element $t$. The proportionality coefficient is $s^{(0)}=x^{(0)} / R_{f}\left(x^{(0)}\right)=10.16 \mathrm{~mm} / 4.517 \mathrm{nH}=2.249 \mathrm{~mm}$ $\cdot \mathrm{nH}^{-1}$. Thus, we have $x^{(1)}=x^{(0)}+s^{(0)} \cdot\left(t_{1}^{(0)}-t_{0}^{(0)}\right)$, which gives $x^{(1)}=14.51 \mathrm{~mm}$. The fine model response at $x^{(1)}$ obtained by Sonnet $\boldsymbol{e m}$ is $6.51 \mathrm{nH}$, which is already acceptable. After another two iterations, tuning space mapping gives $x^{(3)}=14.48 \mathrm{~mm}$; the corresponding line inductance is again $6.50 \mathrm{nH}$.

Type 1 Embedding With Indirect Tuning Parameter: See Fig. 6(d). After initial matching, $t_{0}^{(0)}=2.74 \mathrm{nH}$. After optimization of the tuning model, we have $t_{1}^{(0)}=4.70 \mathrm{nH}$.

For the calibration step, we use a linear dependence between the microstrip length $x$ and the inductance of the tuning element $t$. The proportionality coefficient is $s^{(0)}=\left(x^{(1)}-x_{1}-\right.$ $\left.x_{2}\right) / t_{0}^{(0)}=3.14 \mathrm{~mm} \cdot \mathrm{nH}^{-1}$. Thus, we have $x^{(1)}=x^{(0)}+s^{(0)}$. $\left(t_{1}^{(0)}-t_{0}^{(0)}\right)$, which gives $x^{(1)}=14.56 \mathrm{~mm}$. The fine model response at $x^{(1)}$ obtained by Sonnet em is $6.54 \mathrm{nH}$. After another two iterations, tuning space mapping gives $x^{(3)}=14.48 \mathrm{~mm}$; the corresponding line inductance is again $6.50 \mathrm{nH}$.

Table II summarizes the first iterations of our illustration.

\section{A. Coupled Microstrip Line}

We demonstrate our tuning method in the 2-D design of a coupled microstrip line using Agilent Momentum. We create the structure with internal ports P3, P4, P5, and P6, as in Fig. 7. The length of the coupled lines is 105 mil and the gap between the lines is 5 mil. We obtain a six-port $S$-parameter file from Agilent Momentum. To obtain the corresponding two-port $S$-parameters, we import the $S$-parameter file to Agilent ADS and connect the adjacent port pairs, as shown in Fig. 8 .

Our tuning model is based on Fig. 8 (representation of the fine model in ADS). We leave open ports 5 and 6 and insert an equivalent-circuit microstrip coupled line MCLIN (Type 1

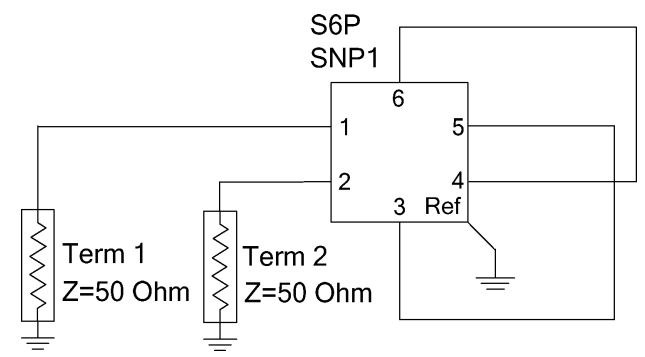

Fig. 8. Importing the six-port Agilent Momentum coupled microstrip line model into Agilent ADS. The adjacent internal port pairs are connected to obtain its corresponding two-port $S$-parameters.

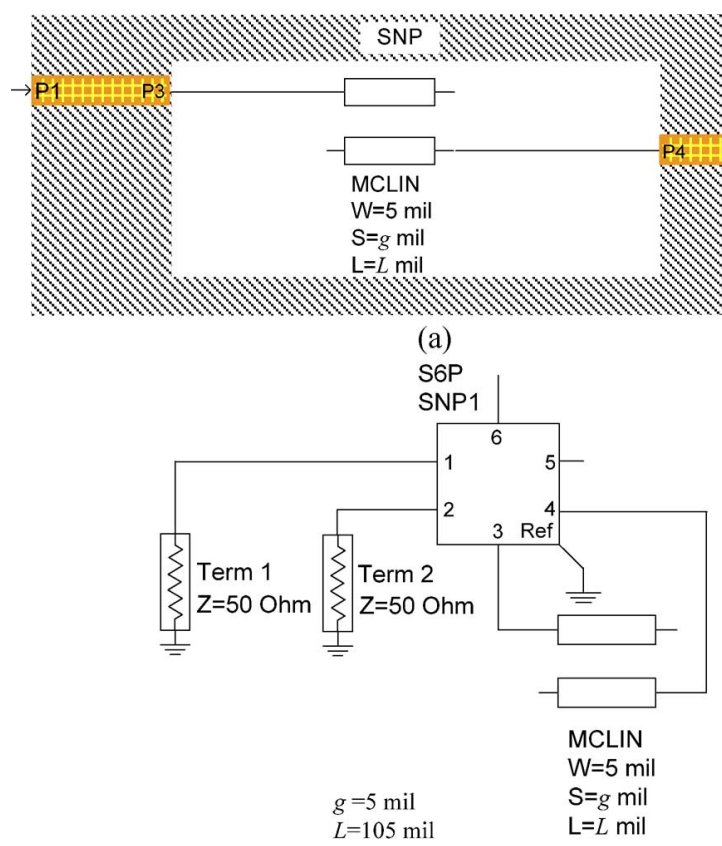

(b)

Fig. 9. Tuning model: an equivalent-circuit coupled microstrip line (Type 1 embedding with direct tuning element) replaces the portion of design interest. (a) Illustration of the tuning model; the hatched area marks what remains in the SNP file. (b) Agilent ADS implementation.

embedding) between ports 3 and 4 and obtain the tuning model shown in Fig. 9.

In the alignment process, we calibrate the dielectric constant and substrate height of the inserted coupled line MCLIN such that the two-port $S$-parameters of the tuning model match that of the fine model. After alignment calibration, we obtain 10.14 mil for the substrate height and 10.10 for the dielectric constant (originally 10 mil and 9.9, respectively). The match between the fine model and tuning model is good. For illustrative purposes, we select our target as an Agilent Momentum $S$-parameter response $(1-10 \mathrm{GHz})$ for the structure shown in Fig. 10. We optimize the surrogate of Fig. 9 to match this target and obtain a good estimate of the design parameter values as $g=10.15$ mil and $L=80.13$ mil in one iteration.

Note that in Type 1 embedding, if the piece being replaced is the entire structure of the fine model (i.e., whole structure between ports 1 and 2 in Fig. 7), the tuning model becomes a standard coarse model and tuning space mapping becomes standard space mapping. 


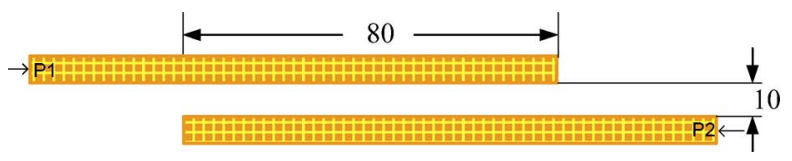

Fig. 10. Coupled microstrip line in Agilent Momentum as our target.

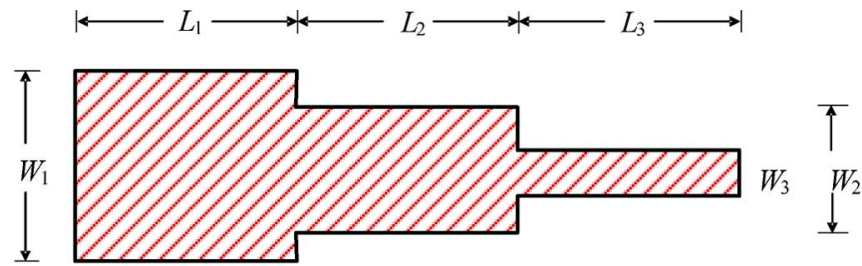

(a)

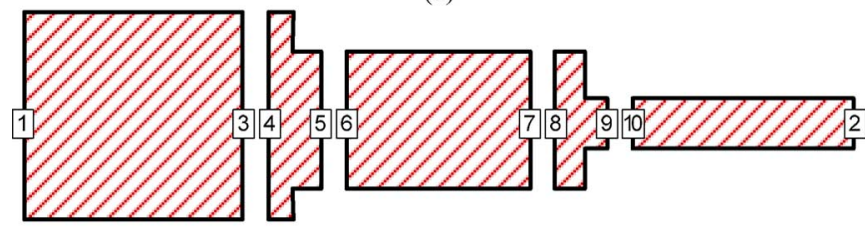

(b)

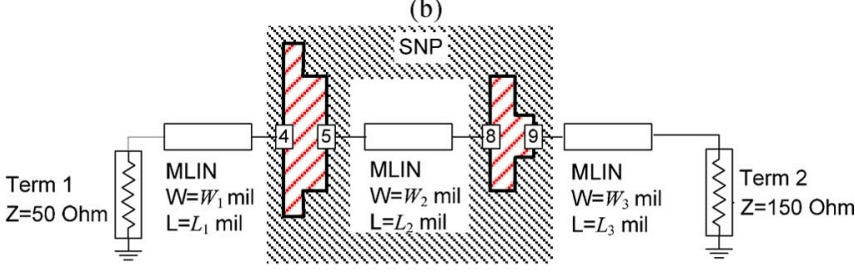

(c)

Fig. 11. Three-section 3:1 microstrip impedance transformer. (a) Structure and dimensions. (b) Sonnet $\boldsymbol{e m}$ model with co-calibrated ports. (c) Tuning model: replacing the designable components with the ADS model. The hatched area marks what remains in the SNP file.

\section{B. Three-Section Transformer Example}

In this example, we demonstrate that our method is capable of designing structures that involve cross-sectional parameters, in this case, widths. This was not easily implemented by the method described in [12] and [13].

Here we demonstrate a three-section transformer example that involves the design of physical widths and lengths, as shown in Fig. 11(a). The design specifications are

$$
\left|S_{11}\right| \leq-20 \mathrm{~dB} \text { for } 5 \mathrm{GHz} \leq \omega \leq 15 \mathrm{GHz}
$$

The model is simulated using Sonnet $\boldsymbol{e m}$. We insert co-calibrated ports inside the structure, as demonstrated in Fig. 11(b). The ten-port $S$-parameter S10P file is then imported into ADS. The ADS microstrip transmission line models replace the sections between ports 1 and 4 , between 5 and 8 , and between 9 and 2, as in Fig. 11(c) in the form of Type 1 embedding with direct tuning elements. This should be distinguished from inserting disconnected fine model segments into an equivalent circuit. The tuning model in the ADS schematic is then calibrated against the fine model response by optimizing the preassigned parameters, i.e., the dielectric constants and substrate heights of the microstrip lines. In this example, each tuning element is assigned an independent set of preassigned parameters. After

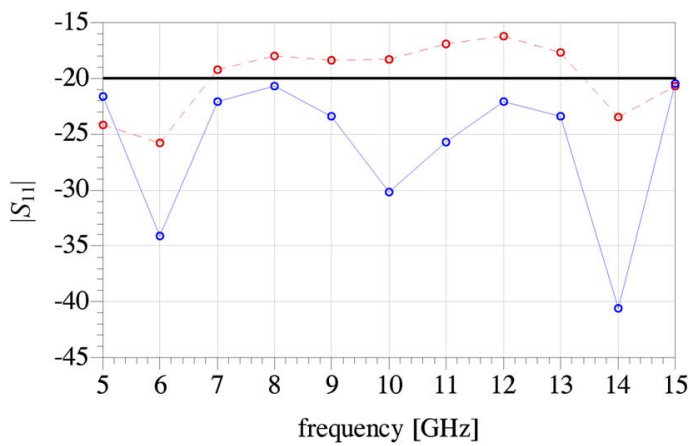

Fig. 12. Three-section 3:1 microstrip impedance transformer Sonnet $\boldsymbol{e m}$ responses: initial $(\ldots \circ \cdots)$ and final $(-\circ-)$ after two iterations.

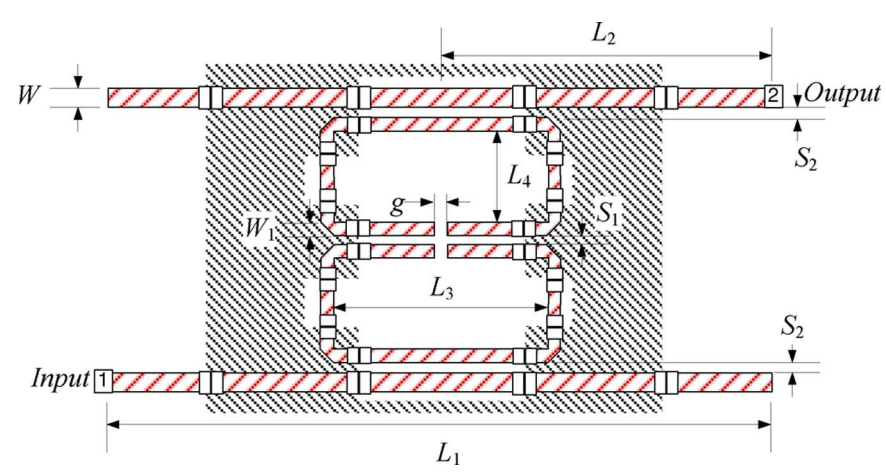

Fig. 13. Open-loop ring resonator bandpass filter: physical structure with co-calibrated ports. Structures outside the hatched area are to be replaced by Type 1 embedding with direct tuning elements.

the model is calibrated, we keep the preassigned parameters as constants and optimize the model in Fig. 11(c) to obtain the next predicted values of the design parameters. In just two iterations, we obtain a good result, as in Fig. 12.

\section{Open-Loop Ring Resonator Bandpass Filter}

Our third example is the open-loop ring resonator bandpass filter [18] shown in Fig. 13. Here, the design parameters are $\boldsymbol{x}=\left[\begin{array}{lllllll}L_{1} & L_{2} & L_{3} & L_{4} & S_{1} & S_{2} & g\end{array}\right]^{T} \mathrm{~mm}$. Other parameter values are $W=0.6 \mathrm{~mm}$ and $W_{1}=0.4 \mathrm{~mm}$. The design specifications are

$$
\begin{aligned}
& \left|S_{21}\right| \geq-3 \mathrm{~dB} \text { for } 2.8 \mathrm{GHz} \leq \omega \leq 3.2 \mathrm{GHz} \\
& \left|S_{21}\right| \leq-20 \mathrm{~dB} \text { for } 1.5 \mathrm{GHz} \leq \omega \leq 2.5 \mathrm{GHz} \\
& \left|S_{21}\right| \leq-20 \mathrm{~dB} \text { for } 3.5 \mathrm{GHz} \leq \omega \leq 4.5 \mathrm{GHz} .
\end{aligned}
$$

In this example, the fine model is simulated in Sonnet $\boldsymbol{e m}$, the tuning model is constructed and optimized in Agilent ADS. To construct the initial tuning model, in Sonnet $\boldsymbol{e m}$, we first divide the microstrip structure and insert co-calibrated port pairs on the cut edges, as shown in Fig. 13. The EM structure with the ports is then simulated and the resulting SNP data file (50 ports) is imported into the SNP $S$-parameter file component in ADS. Equivalent-circuit microstrip lines and coupled line components replace sections of the physical structure in Fig. 14. A new tuning model is now available in the ADS circuit simulator. The design parameters are the same as those of the original de-

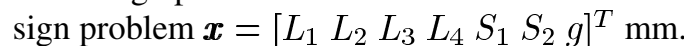

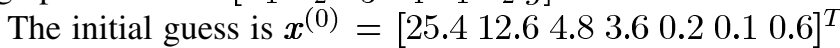
$\mathrm{mm}$. We show the response of our tuning model, fine model, 


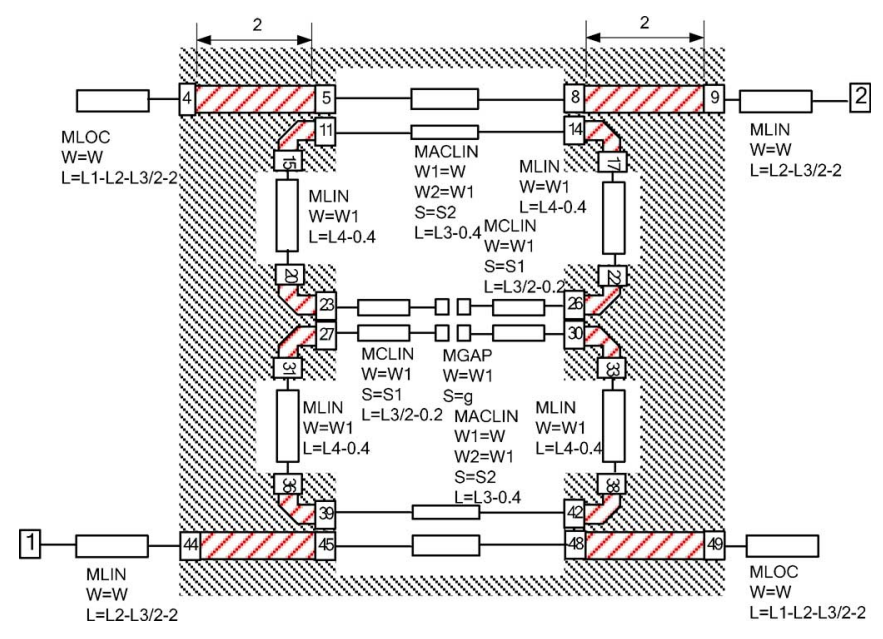

Fig. 14. Open-loop ring resonator bandpass filter in ADS using Type 1 embedding with direct tuning elements.

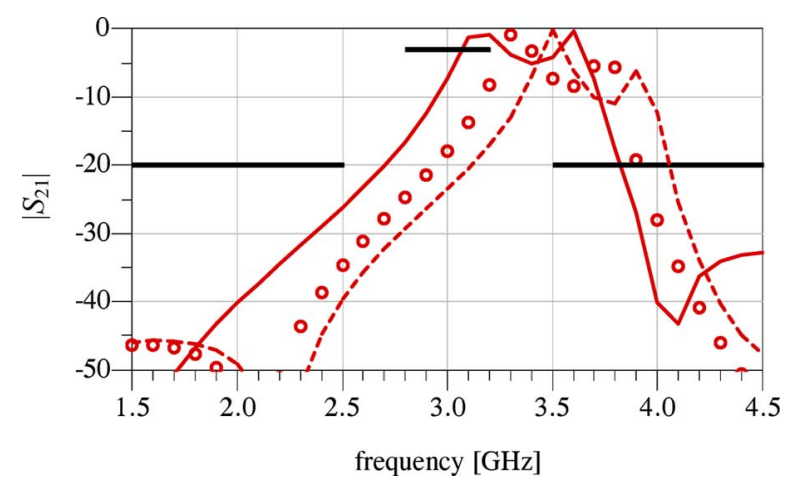

Fig. 15. Initial responses: tuning model (-), fine model (o), and fine model with co-calibrated ports (- - -).

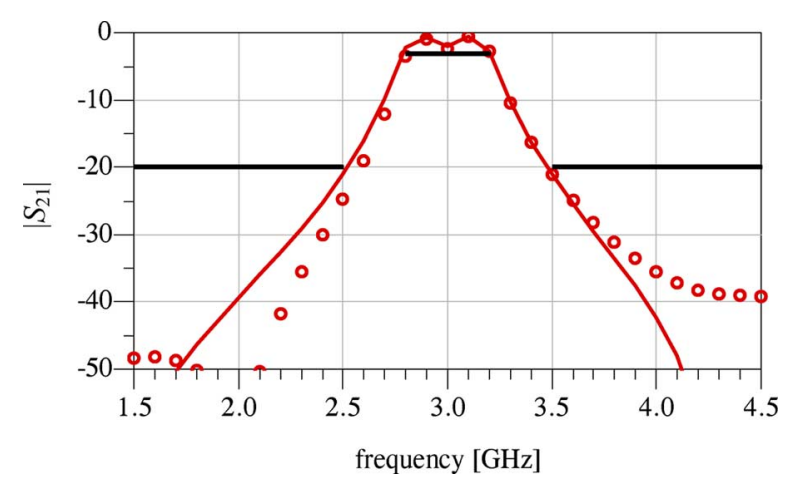

Fig. 16. Responses after two iterations: the tuning model (-) and corresponding fine model $(\mathrm{o})$.

and fine model with co-calibrated ports in Fig. 15. We compensate the deviation between the tuning model and the fine model by calibrating the preassigned parameters, dielectric constant, and substrate height of the tuning elements. In this example, all tuning elements share one set of preassigned parameters in the calibration process.

The tuning model is optimized in ADS with respect to the design parameters. The new design parameters are then assigned to the fine model. The optimal values obtained with (3) are

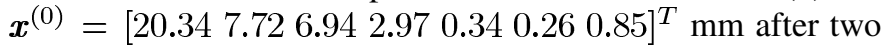

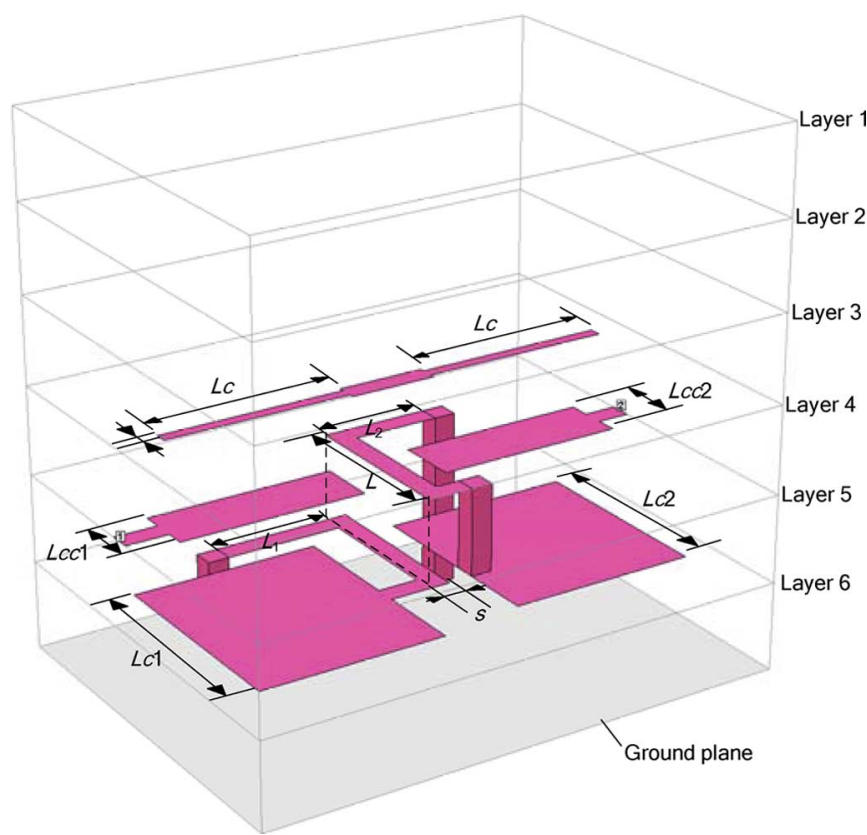

Fig. 17. LTCC second-order bandpass filter [19].

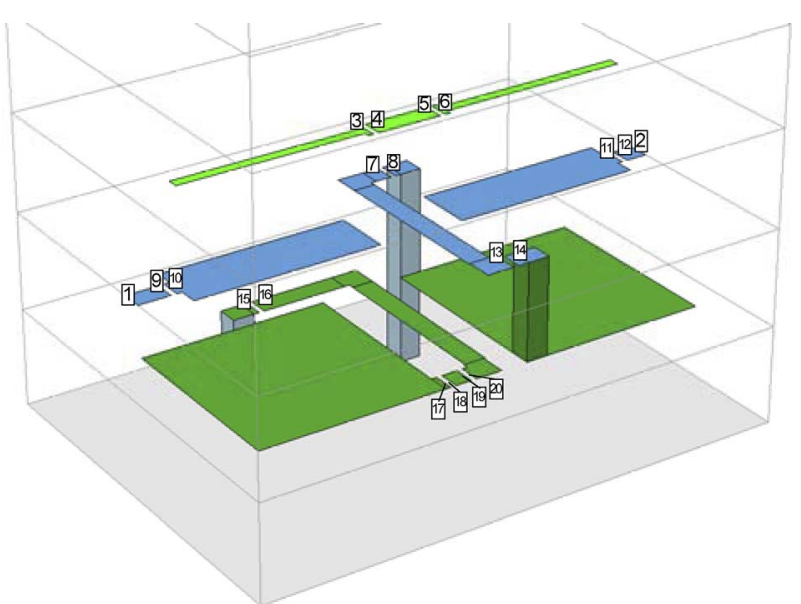

Fig. 18. LTCC second-order bandpass filter with co-calibrated ports.

iterations. The optimized tuning model and the corresponding fine model responses are shown in Fig. 16.

\section{LTCC Second-Order Bandpass Filter}

We design a compact low-temperature co-fired ceramic (LTCC) lumped-element second-order bandpass filter [19]. The filter is shown in Fig. 17. The multilayer LTCC structure is not easily designable before our introduction of Type 1 embedding.

The design parameters are $x=$ $\left[\begin{array}{lllllllll}L_{c} & L & L_{c c 1} & L_{c c 2} & L_{c 1} & L_{c 2} & L_{1} & L_{2} & s\end{array}\right]^{T}$ mil and the specifications are

$$
\begin{aligned}
& \left|S_{21}\right| \leq-20 \text { for } 1 \mathrm{GHz} \leq \omega \leq 2 \mathrm{GHz} \\
& \left|S_{21}\right| \leq-20 \text { for } 3 \mathrm{GHz} \leq \omega \leq 4 \mathrm{GHz} \\
& \left|S_{21}\right| \geq-2 \text { for } 2.35 \mathrm{GHz} \leq \omega \leq 2.65 \mathrm{GHz}
\end{aligned}
$$

In order to plug in tuning elements, we add 18 co-calibrated ports to the structure (Fig. 18). 


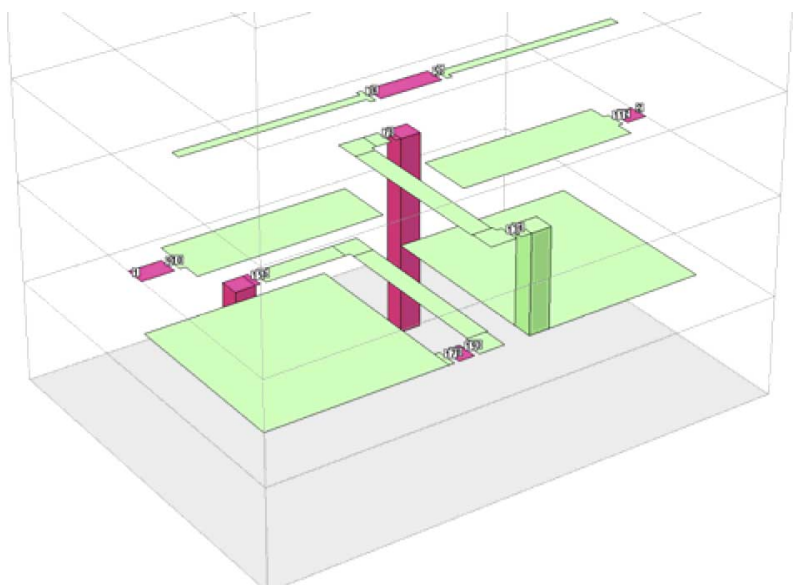

Fig. 19. LTCC second-order bandpass filter: the green (in online version) (or light) areas are to be replaced by Type 1 embedded tuning elements.

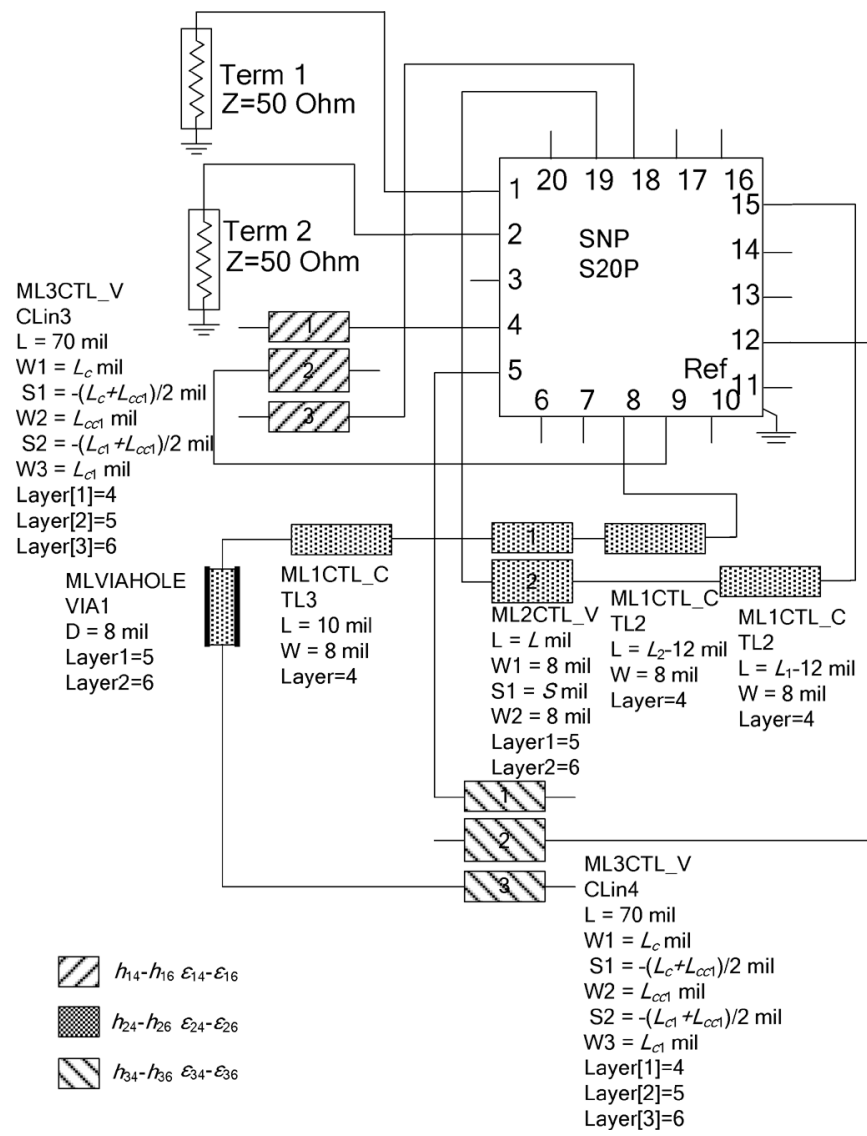

Fig. 20. Tuning model of the LTCC second-order bandpass filter in Agilent ADS is ready to be designed.

We now import the 20-port $S$-parameter file S20P into Agilent ADS and replace the green area (in online version) with Type 1 embedded tuning elements (Fig. 19). Since the couplings between the layers have to be taken into consideration, we use multilayer coupled lines as tuning elements to replace the coupled multilayer structure.

The tuning model (Fig. 20) in Agilent ADS is ready to be optimized. We apply our tuning space mapping algorithm. We extract initial tuning parameters $\boldsymbol{t}_{0}$ and preassigned parameters $\boldsymbol{x}_{p}$ in the alignment process. The selected preassigned parameters are the dielectric constants and substrate heights

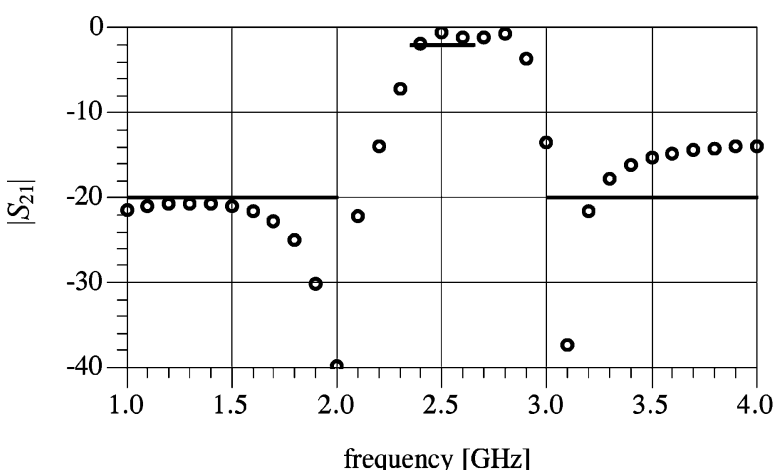

(a)

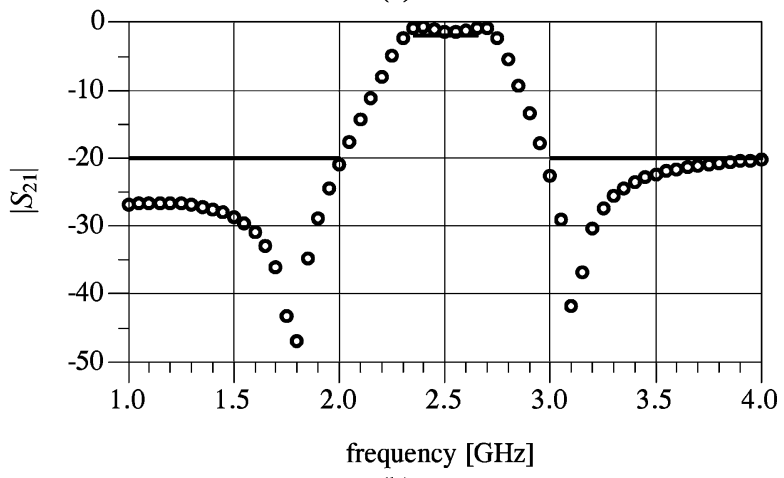

(b)

Fig. 21. LTCC second-order bandpass filter responses at the: (a) initial design and (b) final design.

of the substrate materials between the nonempty layers. They are not uniformly varied in all the components. We divide the tuning elements into three groups, which are shaded by different patterns shown in Fig. 20. Each group has its own multilayer substrate property values that can be varied during the alignment process. The initial guess of the design param-

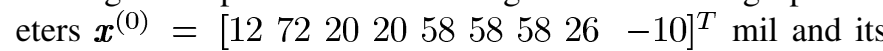
corresponding response is shown in Fig. 21(a). In four iterations, we obtain a good fine model response as in Fig. 21(b)

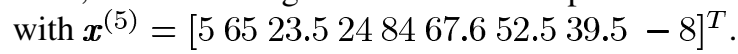

\section{DISCUSSION}

Performance-wise, our tuning approach is similar to our previous space mapping approach in that the optimization process is completed in just two or three iterations with one or two fine model simulations in each iteration. However, tuning space mapping may save setup time and effort for some engineers since it generally does not need a full-system coarse model as a base for building the surrogate. The tuning model or surrogate is built on an existing fine or EM model.

\section{CONCLUSION}

We present a tuning space mapping framework featuring physically based tuning elements to facilitate simulator-based tuning design. Our new tuning space mapping framework consolidates and simplifies the approach described in [13]. The framework offers flexibility in choosing between direct and indirect tuning elements and between our so-called Type 0 and Type 1 embedding. It is accurate since the tuning model is embedded with fine-model couplings and discontinuity information, and aligned with the fine model. The tuning 
elements facilitate direct or (simply calibrated) indirect access to the physical design parameters. Our procedure makes a larger variety of library circuit-based tuning elements usable. It effectively solves the problem of cross-sectional parameter design.

\section{ACKNOWLEDGMENT}

The authors thank Sonnet Software Inc., Syracuse, NY, for em, and Agilent Technologies, Santa Rosa, CA, for making ADS and Momentum available. The authors also thank Dr. J. C. Rautio for useful ongoing discussions.

\section{REFERENCES}

[1] R. V. Snyder and S. Shin, "Parallel coupled line notch filter with wide spurious-free passbands," in IEEE MTT-S Int. Microw. Symp. Dig., Long Beach, CA, Jun. 2005, pp. 253-256.

[2] R. V. Snyder, "Practical aspects of microwave filter development," IEEE Microw. Mag., vol. 8, no. 2, pp. 42-54, Apr. 2007.

[3] S. Shin and S. Kanamaluru, "Diplexer design using EM and circuit simulation techniques," IEEE Microw. Mag., vol. 8, no. 2, pp. 77-82, Apr. 2007.

[4] S. Shin, "Co-simulation techniques using 3D EM and circuit simulators for microwave filter implementations," presented at the IEEE MTT-S Int. Microw. Symp. Workshop, Long Beach, CA, Jun. 2005.

[5] V. Rizzoli, A. Costanzo, D. Masotti, and P. Spadoni, "Circuit-level nonlinear/electromagnetic co-simulation of an entire microwave link," in IEEE MTT-S Int. Microw. Symp. Dig., Long Beach, CA, Jun. 2005, pp. 813-816.

[6] J. Sercu and F. Demuynck, "Electromagnetic/circuit co-optimization of lumped component and physical layout parameters using generalized layout components," in IEEE MTT-S Int. Microw. Symp. Dig., Seattle, WA, Jun. 2002, pp. 2073-2076.

[7] A. Bhargava, "Designing circuits using an EM/circuit co-simulation technique," RF Design, p. 76, Jan. 2005.

[8] D. G. Swanson and R. J. Wenzel, "Fast analysis and optimization of combline filters using FEM," in IEEE MTT-S Int. Microw. Symp. Dig., Boston, MA, Jul. 2001, pp. 1159-1162.

[9] D. Swanson and G. Macchiarella, "Microwave filter design by synthesis and optimization," IEEE Microw. Mag., vol. 8, no. 2, pp. 55-69, Apr. 2007.

[10] J. C. Rautio, "RF design closure-Companion modeling and tuning methods," presented at the IEEE MTT-S Int. Microw. Symp. Workshop, San Francisco, CA, 2006.

[11] J. C. Rautio, "Perfectly calibrated internal ports in EM analysis of planar circuits," in IEEE MTT-S Int. Microw. Symp. Dig., Atlanta, GA, Jun. 2008, pp. 1373-1376.

[12] J. Meng, S. Koziel, J. W. Bandler, M. H. Bakr, and Q. S. Cheng, "Tuning space mapping: A novel technique for engineering design optimization," in IEEE MTT-S Int. Microw. Symp. Dig., Atlanta, GA, Jun. 2008, pp. 991-994.

[13] S. Koziel, J. Meng, J. W. Bandler, M. H. Bakr, and Q. S. Cheng, "Accelerated microwave design optimization with tuning space mapping," IEEE Trans. Microw. Theory Tech., vol. 57, no. 2, pp. 383-394, Feb. 2009.

[14] Q. S. Cheng, J. W. Bandler, and S. Koziel, "Tuning space mapping optimization exploiting embedded surrogate elements," in IEEE MTT-S Int. Microw. Symp. Dig., Boston, MA, Jun. 2009, pp. 1257-1260.
[15] J. C. Rautio, "EM-component-based design of planar circuits," IEEE Microw. Mag., vol. 8, no. 4, pp. 79-90, Aug. 2007.

[16] J. W. Bandler, Q. S. Cheng, S. A. Dakroury, A. S. Mohamed, M. H. Bakr, K. Madsen, and J. Søndergaard, "Space mapping: The state of the art," IEEE Trans. Microw. Theory Tech., vol. 52, no. 1, pp. 337-361, Jan. 2004.

[17] Q. S. Cheng, J. W. Bandler, and S. Koziel, "Combining coarse and fine models for optimal design," IEEE Microw. Mag., vol. 9, no. 1, pp. 79-88, Feb. 2008.

[18] S. Koziel and J. W. Bandler, "Space mapping with multiple coarse models for optimization of microwave components," IEEE Microw. Wireless Compon. Lett., vol. 8, no. 1, pp. 1-3, Jan. 2008.

[19] L. K. Yeung and K.-L. Wu, "A compact second-order LTCC bandpass filter with two finite transmission zeros," IEEE Trans. Microw. Theory Tech., vol. 51, no. 2, pp. 337-341, Feb. 2003.

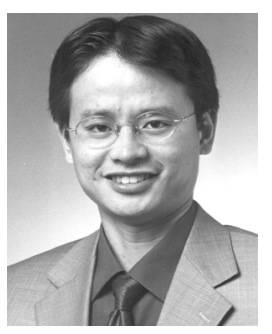

Qingsha S. Cheng (S'00-M'05-SM'09) was born in Chongqing, China. He received the B.Eng. and M.Eng. degrees from Chongqing University, Chongqing, China, in 1995 and 1998, respectively, and the Ph.D. degree from McMaster University, Hamilton, ON, Canada, in 2004.

In 1998, he joined the Department of Computer Science and Technology, Peking University, Beijing, China. In 1999, he joined the Department of Electrical and Computer Engineering, McMaster University, where he is currently a Research Associate with the Department of Electrical and Computer Engineering and a Lecturer with the Faculty of Engineering. His research interests are surrogate modeling, computer-aided design (CAD), modeling of microwave circuits, software design technology, and methodologies for microwave CAD.

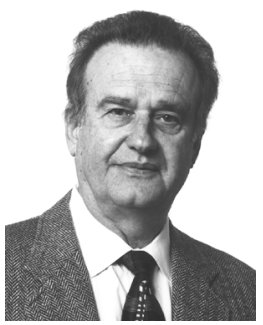

John W. Bandler (S'66-M'66-SM'74-F'78LF'06) studied at Imperial College, London, U.K. He received the B.Sc. (Eng.), Ph.D., and D.Sc. (Eng.) degrees from the University of London, London, U.K., in 1963, 1967, and 1976, respectively.

In 1969, he joined McMaster University, Hamilton, ON, Canada, where he is currently a Professor Emeritus. He was President of Optimization Systems Associates Inc., which he founded in 1983, until November 1997, the date of its acquisition by the Hewlett-Packard Company. He is President of Bandler Corporation, Dundas, ON, Canada, which he founded in 1997.

Dr. Bandler is a Fellow of several societies including the Royal Society of Canada. He was the recipient of the 2004 IEEE Microwave Theory and Techniques Society (IEEE MTT-S) Microwave Application Award.

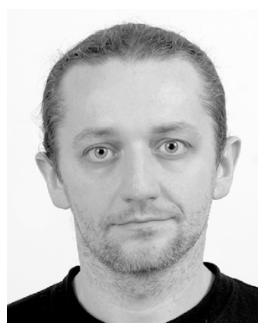

Slawomir Koziel (M'03-SM'07) received the M.Sc. and Ph.D. degrees in electronic engineering from the Gdańsk University of Technology, Gdańsk-Wrzeszcz, Poland, in 1995 and 2000, respectively, and the M.Sc. degree in theoretical physics. M.Sc. degree in mathematics, and Ph.D. degree in mathematics from the University of Gdańsk, Gdańsk, Poland, in 2000, 2002, and 2003, respectively.

He is currently an Associate Professor with the School of Science and Engineering, Reykjavik University, Reykjavik, Iceland. His research interests include surrogate-based modeling and optimization, space mapping, circuit theory, analog signal processing, evolutionary computation, and numerical analysis. 